\title{
Lightning Currents on Fastening Assemblies of an Aircraft Fuel Tank, Part II: FDTD Modeling merged with a Circuit Model supplemented by a Statistical Model
}

\author{
P. Monferran, C. Guiffaut, Member, IEEE, A. Reineix, Member, IEEE, F. Fustin and F. Tristant
}

\begin{abstract}
This paper presents a fastening assemblies FDTD model based on a lumped element circuit integrated into thin wire formalism. It can be used to quantify current dispersion or extreme current in aircraft fuel tank fasteners and to optimize the lightning protection in hotspot zones. An original method allows the characterization of the fastener lumped resistances. This method dealing with DC measurement and an optimization process is validated provided that 1 ) the measurements number is greater than the lumped elements number and 2) each node of the circuit is used at least once. Due to the large uncertainties from one fastener to the other one in the same family, the circuit model is supplement with a statistical model using the results about the fastener resistances state after a lightning shot presented in part I. Stochastic distribution values are applied to each lumped resistance of the fastener circuit model. Making a hybridization between a circuit solver and the FDTD method, the circuit model is added in the same 3D FDTD generic fuel tank modelling as presented in part $I$. Comparing the current distributions of the simple model of the part $I$ and the circuit model, the uncertainties introduced by each model are presented.

Index Terms - Statistical method, finite difference time domain (FDTD) modelling, fasteners modeling, lightning effects , fuel tank aircraft, composite material.
\end{abstract}

\section{INTRODUCTION}

$\mathrm{I}^{\mathrm{s}}$ N PART I of this three-part paper [1], the significance of fasteners in the aircraft lightning effects has been introduced [2], [3]. More specifically, in the confined zone as fuel tanks where the sparking effects around the fasteners can be critical, this issue is monitored following a specific standard [4]. Notwithstanding the protections against sparking effects [5-7], they do not prevent the sparking occurrence but they confine it to avoid the ignition of the fuel vapor. As a consequence, the importance of the understanding of the sparking effects has been highlighted in the part I. Moreover, according to [4], the knowledge of the current distributions through the aircraft is needed in order to protect the fuel

The writing of this paper was supported by the XLIM Laboratory.

P. Monferran, C. Guiffaut and A. Reineix are with Xlim laboratory, 123 avenue Albert Thomas, 87000 Limoges, France (phone: +33-5-55-45-77-39; fax: +33-5-55-45-77-66; e-mail : paul.monferran@xlim.fr, christophe.guiffaut@xlim.fr and alain.reineix@xlim.fr)

F. Fustin and F. Tristant are with Dassault Aviation Company, 78 Quai Marcel Dassault, 92552 Saint-Cloud, France (e-mail: fabian.fustin@dassaultaviation.com and fabrice.tristant@dassault-aviation.com). system and to optimize the fasteners number when mechanical constraints are alleviated. Few fastener models have been developed as circuit model [8] or still 3D modelling [4]. Whatever the fastener model, one of the main parameters is the contact resistances in the lightning bandwidth. Most of the time, the uncertainties about the values of these resistances are ignored due to the difficulty to develop a characterization method dealing with measurement. The engineers are compelled to use nominal resistances values by fastener class estimated around few milliohms [3], [9], [10] in their fastener models. A method based on statistical analysis for taking into account these uncertainties has been presented in [1]. It allows us to define a probability law for the equivalent resistance of a fastener whatever the type of this one. This law has been established only for the fasteners which have been subjected to a lightning injection. This result has been easily used to supplement the simple fastener model (SM) built with a wire and a resistance. Nevertheless, if the fastener model is composed by several resistances as in [3] and [8], the statistical model cannot be directly applied.

This paper presents a new fastener model. This model is a lumped resistance circuit model (CM) introduced in a homemade Finite Difference Time Domain (FDTD) software (TEMSI-FD [11]). This implementation is integrated in the oblique thin wire formalism [12] proposed for the FDTD method. In that one, a lumped resistance is usually located in the middle of the segment as the in-cell inductance. Instead, we propose a general approach to connect circuit ports to wire ends. The presented lumped resistance circuit model is built for one of the most complicated fastener used by DassaultAviation including geometrical and electrical criteria of a specific fastening assembly (see Fig. I- 1$)^{2}$. In addition, an original method dealing with DC measurement and an optimization process in the circuit model is presented in order to recover each contact resistance of the circuit model from multipoint measurements of impedances. Some particular conditions are established to apply the proposed method. In the context of a stochastic FDTD modelling as in part I, a statistical model is used. An optimized probability law can be applied for each lumped resistance such as the equivalent resistance of the CM follows the probability law defined in part I. This optimized probability law is obtained assuming

\footnotetext{
${ }^{2}$ Henceforth, equations, figures, or sections from Part $\mathrm{I}$ in [1] are referred to using the prefix I, e.g., Fig. I-1 or Eq. (I-1).
} 
that each lumped resistance of the $\mathrm{CM}$ follows the same probability law. The optimization process of the probability law is split into two parts. First, using the response surface method (RSM), a probability law with a good general statistical behavior is defined. Then, to enhance in particular the right-tail of this probability law, a clearly justified statistical method is proposed. As in the part I, this result is used to supplement the CM, and a stochastic study is made about the current distribution in a simplified fuel tank FDTD modelling. This application allows us to compare our models from a statistical point of view in order to determine their impact for a metallic and a composite fuel tank modelling. The aim of this paper is clearly to highlight the influence of the two different models. In particular, the connections between two fasteners are represented in the CM. Their influence in the current distribution is an important question for the EMC community of the aeronautical field [13], [14].

First, the CM is presented in section II, describing its building, the characterization method of the resistances values, the numerical validation of this method, and the implementation of the CM in the FDTD method. In the section III, the method for building a statistical model according to the one presented in part I is carried out. Finally, we make a comparison between the two fastener models deduced from a stochastic study of a fuel tank modeled by the FDTD method.

\section{THE LUMPED RESISTANCE CIRCUIT MODEL}

\section{A. Presentation of the lumped resistances circuit model}

The methodology presented in this section can be applied whatever the fastener designs. As explained in section I, we choose one of the most complicated fastener used by DassaultAviation. This choice allows us to highlight the worst case for our methodology. Fig. 1 presents a schematic of this float nut fastener. Fig. 2 shows the CM representation for an assembly of two float nut fasteners as the one represented in Fig. I-2. We decide to follow 3 rules for the CM build:

- each metallic contact is represented by a lumped resistance,

- non-conductive gap and metal losses are considered insignificant in the bandwidth of interest,

- the metallic plates are modeled with a resistance (material parameter) and an inductance (geometrical parameter).

In the following, the metallic plates modelling will not be made. Indeed, the resistance of the metallic plates are very low against the contact resistances. For the same reason, the inductive effect induced by the metallic plates at low frequency can be considered insignificant. The modelling is composed with 6 resistances for each fastener with a link between both at the node $V_{1}$. In order to make the following more understandable, the Table I proposes a numbering system for the resistances.

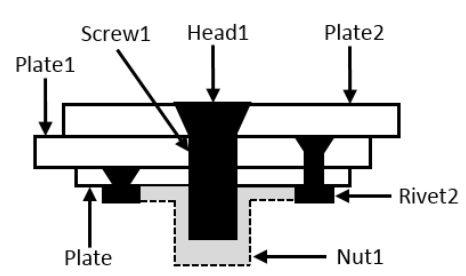

Fig. 1. Float nut fastener illustration.

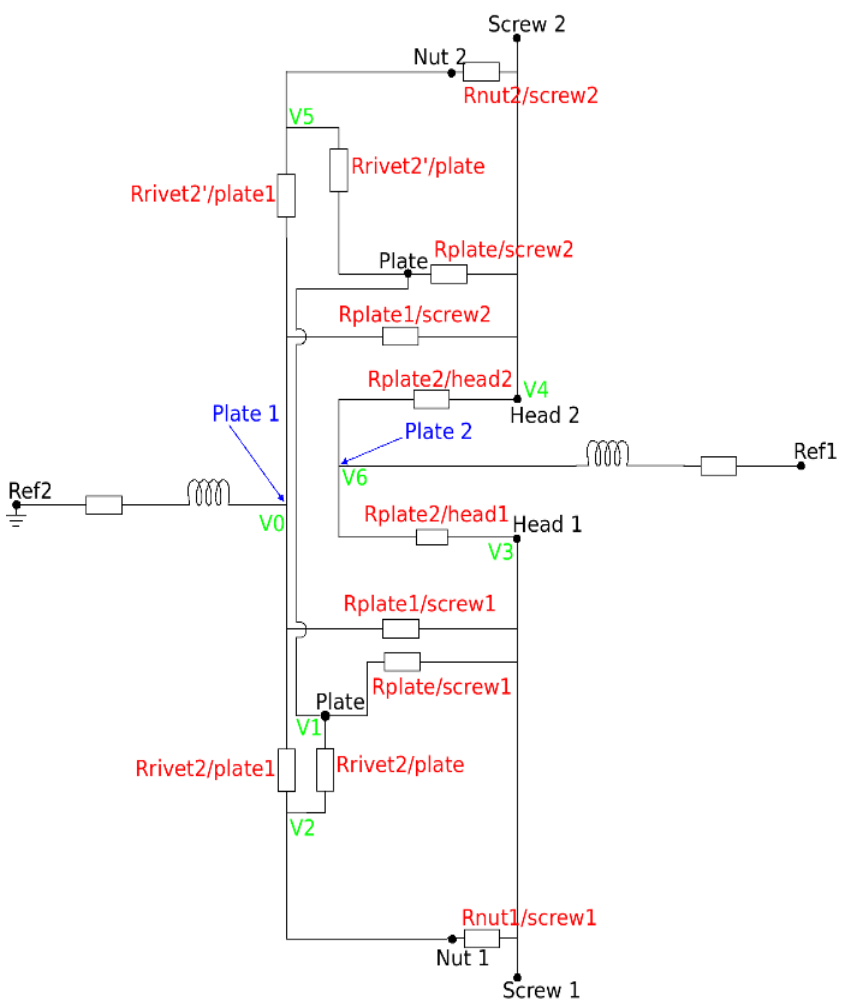

Fig. 2. Lumped resistances circuit model of a two float nut fasteners assembly.

\section{B. Characterization method of the lumped resistances}

In this subsection, we propose an original characterization method of the lumped resistances of the CM dealing with DC measurement and an optimization process requiring the following 4 steps:

1) do $\mathrm{N}$ measures of resistances $R_{n, \text { mes }}$ with a microhmmeter (as the OM16 [15]) between two nodes $\mathrm{i}$ and $\mathrm{j}$ of the circuit,

2) applying the Circuit Nodal Analysis (NA) [16] in the CM with a reference node $V_{0}$, we can write matrix system $[G] \overrightarrow{\mathrm{V}_{n}}=\overrightarrow{\mathrm{I}_{n}}$,

3) then, inverting the conductance matrix $[G]$, the voltage vector is deduced $\overrightarrow{\mathrm{v}_{n}}=[G]^{-1} \overrightarrow{\mathrm{I}}_{n}$,

4) finally, using an optimization algorithm, we minimize the cost function $f=\sum_{n=1}^{N}\left(\frac{V_{n, i}-V_{n, j}}{I_{0}}-R_{n, \text { mes }}\right)^{2}$ for the $\mathrm{N}$ matrix systems.

In our system of equations, we note that $[G]$ is invariant since only the lumped resistances make up this matrix. However, the current vector $I_{n}$, which is composed with the magnitude of the equivalent current source between the two measured nodes, is

TABLE I

LUMPED RESISTANCE NUMBERING SYSTEM

\begin{tabular}{|l|c|c|c|}
\hline $\mathrm{R}_{1}$ & $\mathrm{R}_{\text {rivet2/plate }}$ & $\mathrm{R}_{7}$ & $\mathrm{R}_{\text {rivet2'/plate }}$ \\
\hline $\mathrm{R}_{2}$ & $\mathrm{R}_{\text {plate/screw1 }}$ & $\mathrm{R}_{8}$ & $\mathrm{R}_{\text {plate/screw2 }}$ \\
\hline $\mathrm{R}_{3}$ & $\mathrm{R}_{\text {nut1/screw1 }}$ & $\mathrm{R}_{9}$ & $\mathrm{R}_{\text {nut2/screw2 }}$ \\
\hline $\mathrm{R}_{4}$ & $\mathrm{R}_{\text {rivet2/plate1 }}$ & $\mathrm{R}_{10}$ & $\mathrm{R}_{\text {rivet2'/plate1 }}$ \\
\hline $\mathrm{R}_{5}$ & $\mathrm{R}_{\text {plate1/screw1 }}$ & $\mathrm{R}_{11}$ & $\mathrm{R}_{\text {plate1/screw2 }}$ \\
\hline $\mathrm{R}_{6}$ & $\mathrm{R}_{\text {plate2/head1 }}$ & $\mathrm{R}_{12}$ & $\mathrm{R}_{\text {plate2/head2 }}$ \\
\hline
\end{tabular}




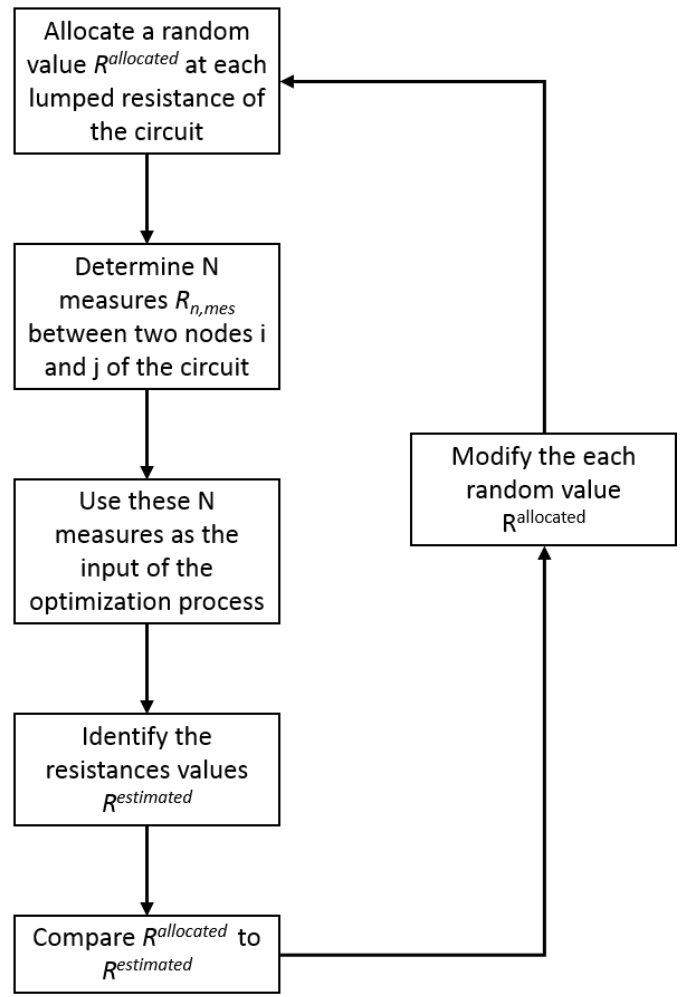

Fig. 3. Flowchart of the numerical validation process.

obviously modified for each measurement. It can be fixed to arbitrary values, for example $\pm 1 \mathrm{~A}$. Only the sign of the current entering in the two adjacent nodes is of importance. For the sake of brevity, we presents only the main characteristics of our optimization algorithm. It uses a Newton descent method based on a gradient calculation and an approximation of the Hessian matrix [17], [18]. This kind of method allows a faster convergence than other global optimization algorithms as genetic ones [19]. The function N2FB of the Port library [20] has been chosen for its flexibility of gradient handling and variable scaling and also for its ability to optimize a large number of variables. The convergence criteria are the standard ones and can be found in [20]. In our case, even with 12 variables, the convergence is

TABLE II

RESULTS OF THE OPTIMIZATION PROCESS PERFORMED 1000 TIMES FOR THE LIMITED CASES (NUMBER OF "MEASURES"=NUMBER OF UNKNOWNS).

\begin{tabular}{|c|c|c|c|c|}
\cline { 2 - 5 } \multicolumn{1}{c|}{} & \multicolumn{2}{c|}{$\begin{array}{c}\text { Case 1 (12 measures } \\
\text { including all nodes) }\end{array}$} & \multicolumn{2}{c|}{$\begin{array}{c}\text { Case 2 (12 measures with 1 } \\
\text { node unused })\end{array}$} \\
\cline { 2 - 5 } \multicolumn{1}{c|}{} & $\Delta E r r_{\text {max }}(\%)$ & $\Delta E r r_{\text {mean }}(\%)$ & $\Delta E r r_{\text {max }}(\%)$ & $\Delta E r r_{\text {mean }}(\%)$ \\
\hline $\mathrm{R}_{1}$ & 200 & 1.5 & 680 & 3.3 \\
\hline $\mathrm{R}_{2}$ & 100 & 0.5 & 58 & 0.6 \\
\hline $\mathrm{R}_{3}$ & 123 & 0.8 & 22 & 15.4 \\
\hline $\mathrm{R}_{4}$ & 60 & 0.2 & 518 & 28.7 \\
\hline $\mathrm{R}_{5}$ & 520 & 1.7 & 520 & 28.6 \\
\hline $\mathrm{R}_{6}$ & 17 & 0.07 & 37 & 0.1 \\
\hline $\mathrm{R}_{7}$ & 70 & 0.5 & 35 & 0.3 \\
\hline $\mathrm{R}_{8}$ & 210 & 1.3 & 52 & 0.2 \\
\hline $\mathrm{R}_{9}$ & 37 & 0.2 & 700 & 16.4 \\
\hline $\mathrm{R}_{10}$ & 148 & 0.4 & 832 & 29.1 \\
\hline $\mathrm{R}_{11}$ & 96 & 0.6 & 834 & 29.1 \\
\hline $\mathrm{R}_{12}$ & 98 & 0.4 & 42 & 0.1 \\
\hline
\end{tabular}

TABLE III

RESULTS OF THE OPTIMIZATION PROCESS PERFORMED 1000 TIMES FOR THE OVERSAMPLED CASES (NUMBER OF "MEASURES">NUMBER OF UNKNOWNS).

\begin{tabular}{|l|c|c|c|c|}
\cline { 2 - 5 } \multicolumn{1}{c|}{} & \multicolumn{2}{c|}{$\begin{array}{c}\text { Case 3 (15 measures } \\
\text { including all nodes) }\end{array}$} & \multicolumn{2}{c|}{$\begin{array}{c}\text { Case 4 (21 measures } \\
\text { including all nodes) }\end{array}$} \\
\cline { 2 - 5 } \multicolumn{1}{c|}{} & $\Delta E r r_{\text {max }}(\%)$ & $\Delta E r r_{\text {mean }}(\%)$ & $\Delta E r r_{\text {max }}(\%)$ & $\Delta E r r_{\text {mean }}(\%)$ \\
\hline $\mathrm{R}_{1}$ & 0.2 & $2.7 \times 10^{-5}$ & 0.2 & $9.5 \times 10^{-6}$ \\
\hline $\mathrm{R}_{2}$ & 0.2 & $3 \times 10^{-5}$ & 0.2 & $1.0 \times 10^{-5}$ \\
\hline $\mathrm{R}_{3}$ & 0.2 & $2.4 \times 10^{-5}$ & 0.2 & $1.4 \times 10^{-5}$ \\
\hline $\mathrm{R}_{4}$ & 0.2 & $3.1 \times 10^{-5}$ & 0.2 & $7.9 \times 10^{-6}$ \\
\hline $\mathrm{R}_{5}$ & 0.2 & $2.7 \times 10^{-5}$ & 0.2 & $7.2 \times 10^{-6}$ \\
\hline $\mathrm{R}_{6}$ & 0.2 & $4.7 \times 10^{-6}$ & 0.2 & $2.6 \times 10^{-6}$ \\
\hline $\mathrm{R}_{7}$ & 0.2 & $8.2 \times 10^{-6}$ & 0.2 & $1.3 \times 10^{-5}$ \\
\hline $\mathrm{R}_{8}$ & 0.2 & $9.1 \times 10^{-6}$ & 0.2 & $8.0 \times 10^{-6}$ \\
\hline $\mathrm{R}_{9}$ & 0.2 & $1.6 \times 10^{-5}$ & 0.2 & $1.4 \times 10^{-5}$ \\
\hline $\mathrm{R}_{10}$ & 0.2 & $2.4 \times 10^{-5}$ & 0.2 & $8.6 \times 10^{-6}$ \\
\hline $\mathrm{R}_{11}$ & 0.2 & $1.5 \times 10^{-5}$ & 0.2 & $8.5 \times 10^{-6}$ \\
\hline $\mathrm{R}_{12}$ & 0.2 & $7.8 \times 10^{-6}$ & 0.2 & $3.1 \times 10^{-6}$ \\
\hline
\end{tabular}

achieved around 20 iterations in less than 1 second.

\section{C.Numerical validation}

As a reminder, the fastener contact resistances are always unknown and any characterization method has been published. As any comparison is possible, we propose a numerical validation of our characterization method based on the preliminary knowledge of the lumped resistance values. The process is presented in Fig. 3. The principle of this validation is to allocate random values $R^{\text {allocated }}$ for each lumped resistance of the $\mathrm{CM}$, then to compute a series of the equivalent resistances between 2 nodes (as we can do a measurement $R_{n, m e s}$ ), and finally to perform the optimization process including these data. At the end of the optimization process, the estimated values $R^{\text {estimated }}$ must be the same as the allocated one at the beginning of the numerical validation. Considering that the convergence time of the optimization process is achieved in less than 1 second, a quasi Monte-Carlo validation is carried out. The numerical validation procedure is performed 1000 times using different set of random resistance values. It takes around 10 seconds. The random resistance values are generated following a uniform distribution in the range from $0.1 \mathrm{~m} \Omega$ to $1 \Omega$. This range is chosen very large in order to verify the efficiency of the optimization process whatever the lumped resistance value.

The CM in Fig. 2 is built including 7 nodes which involve a maximum number of measurements of $C_{7}^{2}=\frac{7 !}{2 !(7-2) !}=21$. Our validation procedure allows us to study several cases with different sets of "measurement". We decide to study 4 cases in order to establish the limitation of the optimization process:

1) A limit case: each set of measurement includes 12 measures using each nodes of the circuit.

2) A limit case without each node used: each set of measurement includes 12 measures using 6 nodes of the circuit ( $V_{0}$ not used).

3) An oversampled case: each set of measurement includes 15 measures using each nodes of the circuit.

4) The ideal case including the 21 measures. 
For each case, we propose to use two estimators in order to compare them. The first one is the maximum error between the allocated value and the estimated one among the 1000 sets of resistances:

$$
\Delta E r r_{\max }=\max \left(\frac{\left|R^{e^{\text {ssimated }}}-R^{\text {allocated }}\right|}{\left|R^{\text {allocated }}\right|} \times 100\right) \text {. }
$$

The second estimator is the mean error between the allocated value and the estimated one of the $n=1000$ sets of resistances noted:

$$
\Delta E r r_{\text {mean }}=\frac{1}{n} \sum_{1}^{n}\left(\frac{\left|R_{i}^{\text {estimated }}-R_{i}^{\text {allocated }}\right|}{\left|R_{i}^{\text {allocated }}\right|} \times 100\right) \text {. }
$$

Both estimators are obviously computed for each lumped resistance.

The Table II presents the results for the both limit cases. It highlights the need to use all nodes. If this condition is not respected, as a matter of fact, we note that the mean error is not acceptable for the lumped resistances related to the unused nodes. Nevertheless, even if all nodes are used, the maximum error can be considered too much important to validate our method as a reliable one.

Our process requires an oversampling of measurement. The Table III presents very good results for the oversampled cases. The errors are sufficiently weak in both cases to highlight the accuracy of the method. For the CM presented in Fig. 2, 15 measures involving all nodes are efficient to estimate the resistances values with a $0.2 \%$ maximum error.

\section{Sensitivity analysis}

The previous sub-section demonstrates numerically that the parameters of the model can be accurately identified provided an over-determined system of equations. However, the proposed inputs are ideal. In order to analyze the sensitivity of the method, we propose to test it with different measurement errors. The OM16 datasheet [15] guarantees a measurement accuracy of $x= \pm 0.05 \%$. Hence, it is the starting point of the sensitivity analysis. The same procedure used in the previous sub-section is performed. Nevertheless, a $x \%$ error is made on each measurement. These measures are given as the input of the optimization process. Fig. 4 presents the results for the case 3 (15 measures) and the case 4 (21 measures). It highlights a high sensitivity of the method against measurement errors. First, it is recommended to include all the possible measurement (case 4). Otherwise, we note that the lack of information combined with a measurement error involves strong estimation errors for some resistances. Typically, the 15 measurement proposed system (case 3) seems to have a lack of equations particularly for the $R_{5}$ optimization. Moreover, accurate measurement is required for an accurate estimation. We estimate the mean accuracy at $5 \%$ for a $1 \%$ measurement error including all nodes.

\section{E. Implementation of a CM in the FDTD method}

In this subsection, we propose a general approach to implement in the FDTD method a circuit model as the one in Fig. 2. An hybridization between TEMSI-FD and an OpenSource software, as NGspice [21] for instance, could be a good way. Nevertheless, we choose to develop our own simple solver in order to have a better integration and portability. The aim is to make several electrical connections from the circuit to the meshed structure using the oblique thin wires formalism [12]. In a CM, different ports can be defined, they allow us to connect one or several wires. The need to manage these connections with the oblique wire formalism is another crucial point justifying our choice.

Several ways to do a circuit solver are possible. We decide to use the MNA (Modified Nodal Analysis, [22]) rather than a circuit state variables method (CSVM) for instance. Indeed,
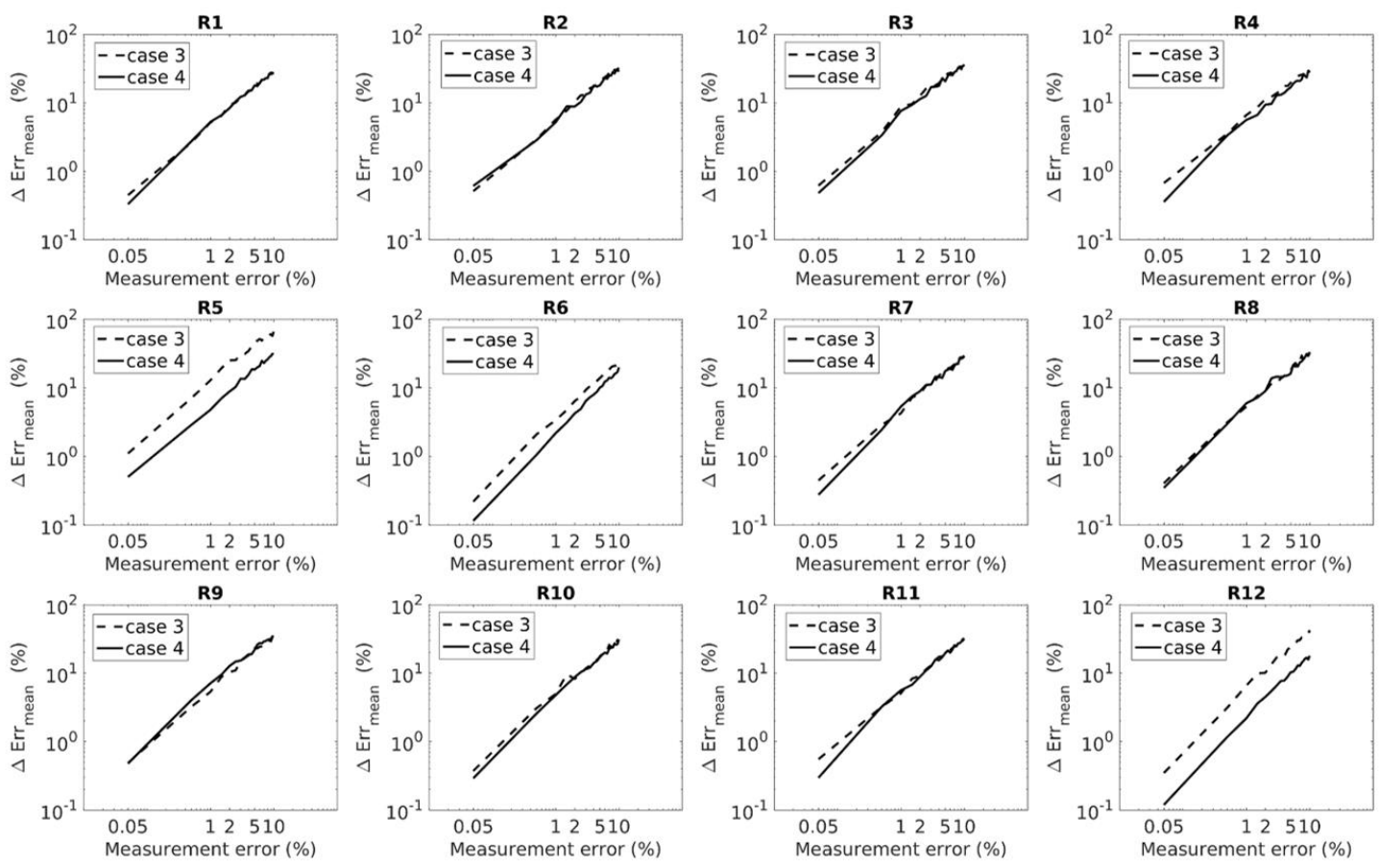

Fig. 4. Sensitivity analysis of the lumped resistances characterization method against measurement errors. 


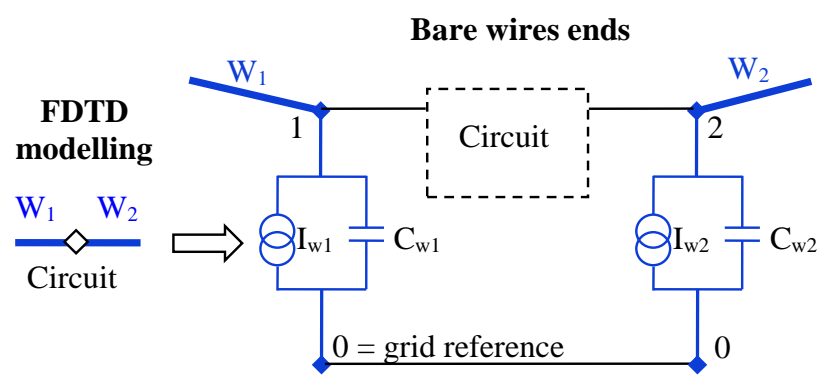

Fig. 5. Hybridization oblique wire formalism with circuit solver.

the systematic build of a circuit with the MNA is a strong benefit in comparison with the CSVM. The MNA linear system to solve can be written as:

$$
[A] \vec{X}=\vec{S} \text {, }
$$

with $\vec{X}$, the unknown vector of node voltages and of branch currents associated to inductance or voltage generator. The vector $\vec{S}$ represents the internal or external (if there is a cable connection) source and $[A]$ is the matrix with the passive circuit elements (resistance, inductance and capacitance). In order to hybrid the MNA solver with the FDTD method, we propose a temporal approach of the circuit solving based on a local implicit Crank-Nicolson (CN) scheme [23]. This scheme is used because of his unconditional stability on the time step. The resulting hybridization, with the oblique wire formalism in the FDTD method, is a stable scheme provided the FDTD Courant-Friedrichs-Lewy (CFL) criterion.

Fig. 5 illustrates the hybridization between the circuit automatically built with the MNA solver and the oblique wire formalism in the FDTD method. Geometrically, the two wire ends are located at the same position in the FDTD modelling in order to maintain the continuity of the current trace in the FDTD grid [12] and therefore avoiding parasitic charge in the $3 \mathrm{D}$ grid. Each connected wire $\left(\mathrm{W}_{1}\right.$ and $\left.\mathrm{W}_{2}\right)$ provides an equivalent current source in parallel with a capacitance, i.e.

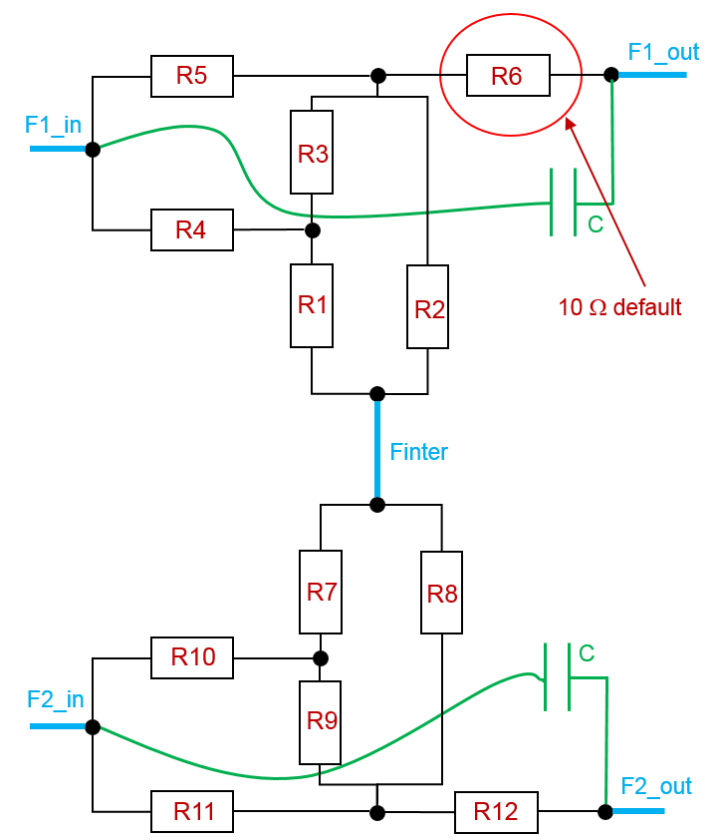

Fig. 6. Lumped resistances circuit model of a two float nut fasteners assembly with capacitors which represent the plates coating.

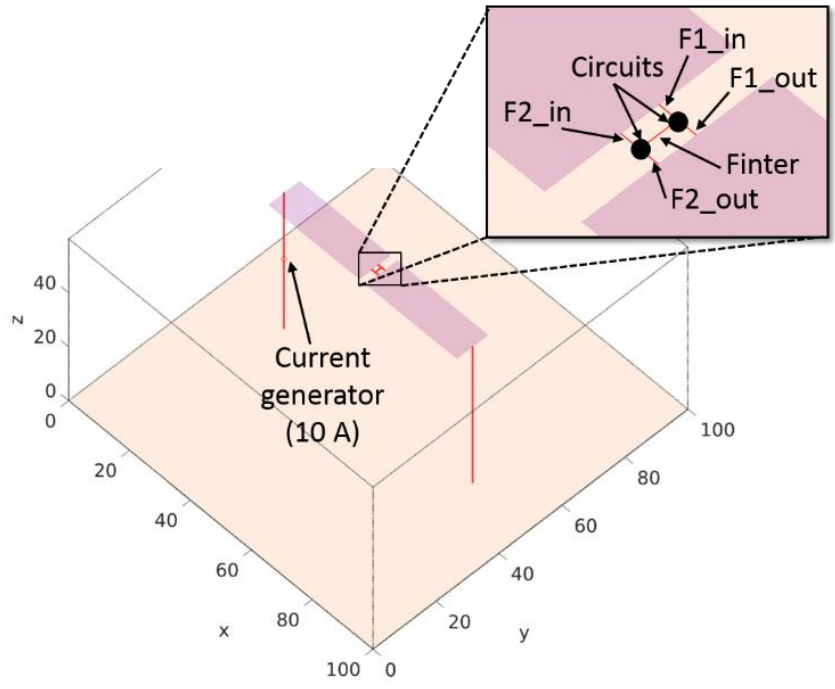

Fig. 7. FDTD modelling of a two float nut fasteners assembly. The axes are in number of mesh.

the in-cell capacitance deduced of the in-cell inductance, connected, in one side to a port of a circuit (nodes denoted 1 or 2), and, in another side to the virtual FDTD grid reference (node denoted 0 ). The voltages between the two nodes returned by the MNA solver are used in the oblique thin wire formalism. Furthermore, we denote that several wires can be connected to the same port. This kind of hybridization allows the inclusion of generators and complex circuits on the nodes of wires in a multiport configuration. That would be really useful for fastener attachment lightning study.

\section{F. Resistive model justification}

The proposed fastener model does not consider parasitic especially capacitors. In this sub-section, we decide to represent the paint sheet which can be represented with a capacitor $C$ in the $\mathrm{CM}$ as shown in Fig. 6. The resistance values are found using the previous proposed method (section II-B) on a real sample. We propose to study a worst case. Hence, we introduce a $10 \Omega$ default on R6. Furthermore, the paint thickness is $10 \mu \mathrm{m}$ instead of $200-1000 \mu \mathrm{m}$ for the usual aircraft paint thickness [24], [25]. Thus, the $C_{\text {square }}$ capacitor can be computed for a $10 \mu \mathrm{m}$ paint thickness with a 10 dielectric constant value:

$$
C_{\text {square }}=\frac{\varepsilon_{0} \varepsilon_{r}}{e}=0.89 \mathrm{nF} / \mathrm{cm}^{2} .
$$

An assembly of two float nut fasteners as the one represented in Fig. I-2 is modelled. Fig. 7 shows the FDTD modelling. A Gaussian waveform is used in the $100 \mathrm{~Hz}-10$

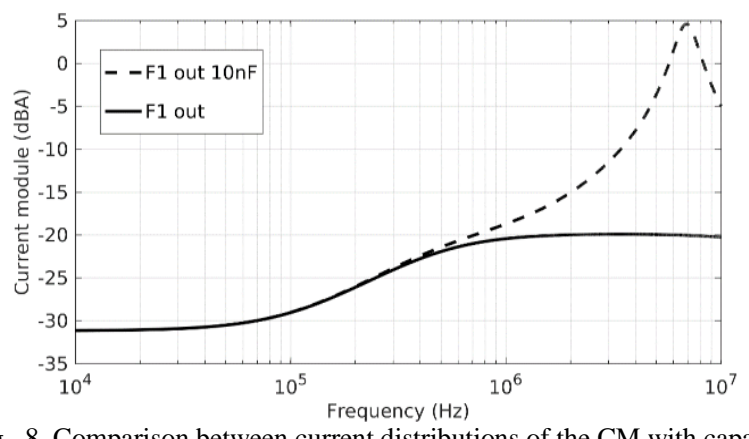

Fig. 8. Comparison between current distributions of the $\mathrm{CM}$ with capacitors (dashe line) and without capacitors (solid line) in function of frequency. 


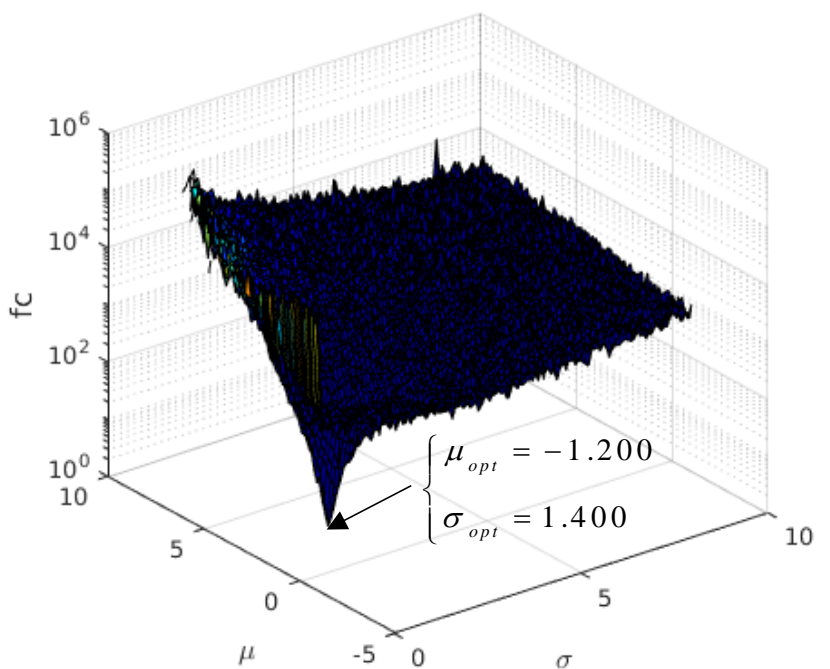

Fig. 9. Illustration of the surface response.

$\mathrm{MHz}$ frequency range. The mesh step is $1 \mathrm{~cm}$. The distance between the two fasteners is two mesh cells. Two simulations are performed: one without capacitors and one with $10 \mathrm{nF}$ capacitors. The value of $10 \mathrm{nF}$ is more than five times the estimated one using (4) for this modelling. Fig. 8 presents the results on the "worst" wire. It highlights that the capacitors have little impact on the current distributions below few megahertz even for this worst case. As a consequence, we assume that a fastener resistive model is adequate for the lightning conduction effects modelling.

\section{STATISTICAL MODEL}

\section{A. Statistical issue}

In part I, we have shown that the equivalent fastener resistances after lightning test shots follow a lognormal law and we have established a model from a measurement campaign database defined by the following parameters $\log N\left(\mu^{\prime}=-1.524, \sigma^{\prime}=0.665\right)$. In order to be consistent with this result, the equivalent resistance of the CM must follow the same lognormal law. Applying a lognormal law $\log N\left(\mu^{c M}, \sigma^{c M}\right)$ for each lumped element of the $\mathrm{CM}$, the equivalent resistance is following a lognormal law

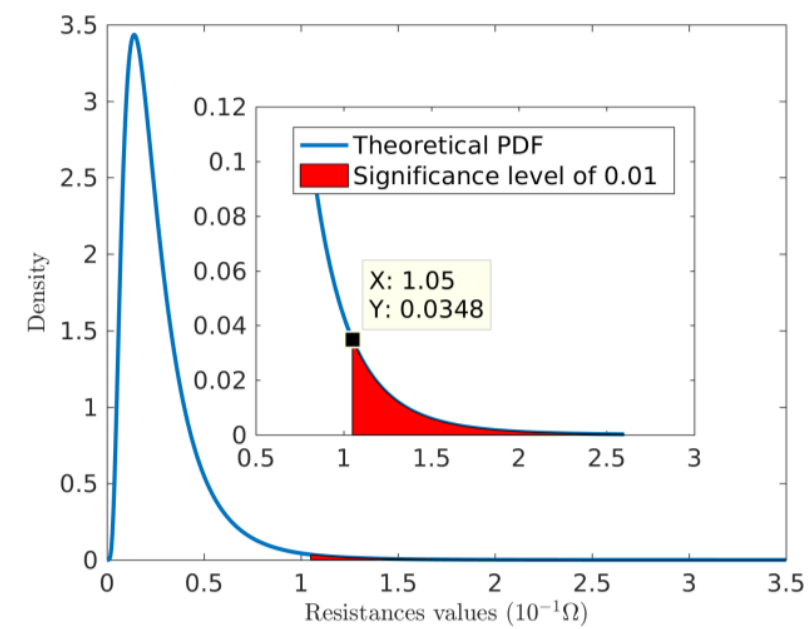

Fig. 11. Theoretical PDF. The inset shows a zoom on the PDF highlighting the area (red) which contains $1 \%$ of the data.

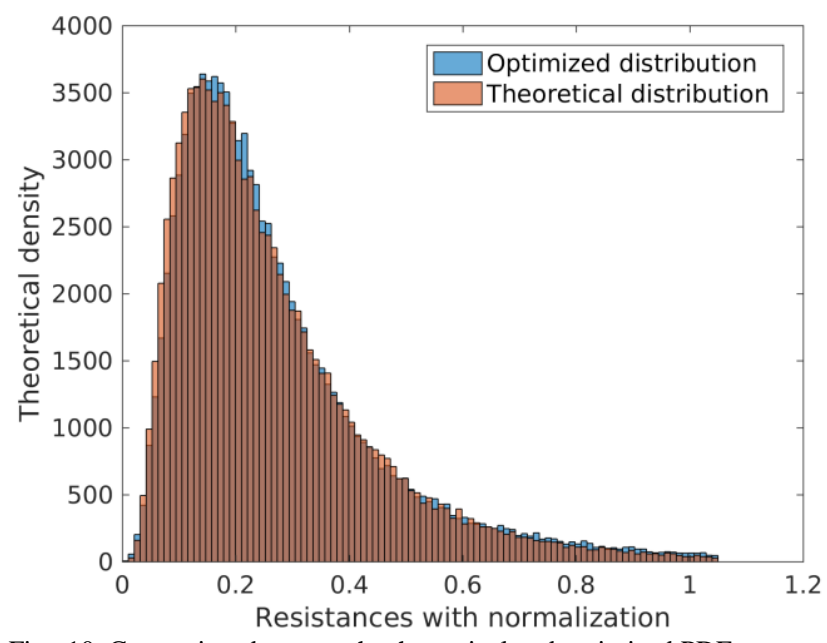

Fig. 10. Comparison between the theoretical and optimized PDF.

$\log N(\mu, \sigma)$. We minimize the difference $f_{c}$ between the theoretical probability distribution function (PDF) of a random set of 100000 samples following $\log N\left(\mu^{\prime}=-1.524, \sigma^{\prime}=0.665\right)$ and the optimized PDF due to the equivalent resistance transformation of the $\mathrm{CM}$ $\log N\left(\mu^{C M}, \sigma^{C M}\right) \rightarrow \log N(\mu, \sigma)$ using the well-known RSM.

This RSM is illustrated in Fig. 9. Applying a lognormal law with $\mu_{o p t}^{C M}$ and $\sigma_{o p t}^{c M}$, the equivalent resistance of the CM exhibits almost the same statistical behavior than the one applied on the SM. Fig. 10 highlights the good agreement between the laws.

\section{B. Statistical distribution enhancement}

Although the agreement seems to be almost perfect between both laws in the Fig. 10, the x-axis normalization hides an important gap between the both standard deviation from the generated random set. We propose an enhancement of the optimized PDF according the mean and standard deviation in order to have a fitting more accurate. The enhancement method is based on bounding conditions and a redistribution of the extreme values. The bounding condition is also applied in the theoretical PDF. We decide to limit the resistance values with a confidence degree of $99 \%$. Plotting the PDF and the

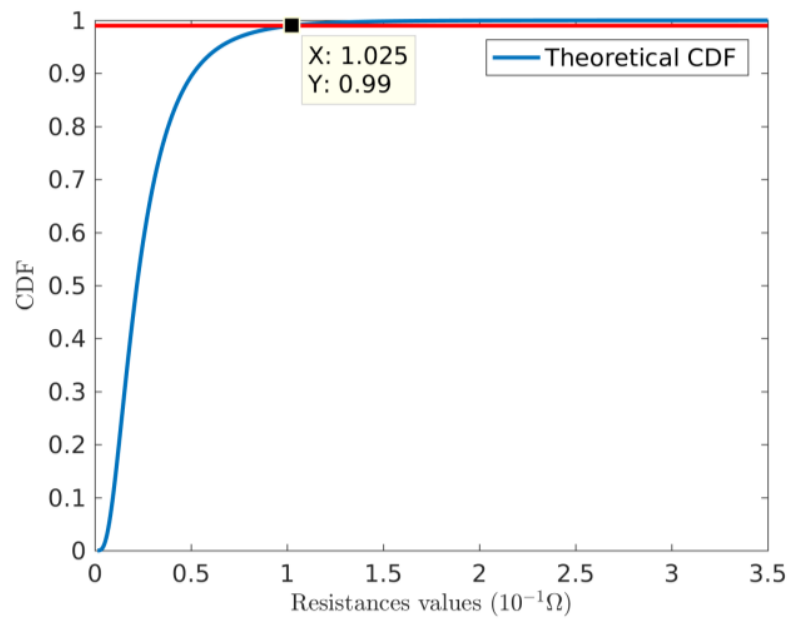

Fig. 12. Theoretical CDF. The intersection between the red line $(y=0.99)$ and the CDF give the limit value where $99 \%$ of the samples are contained. 

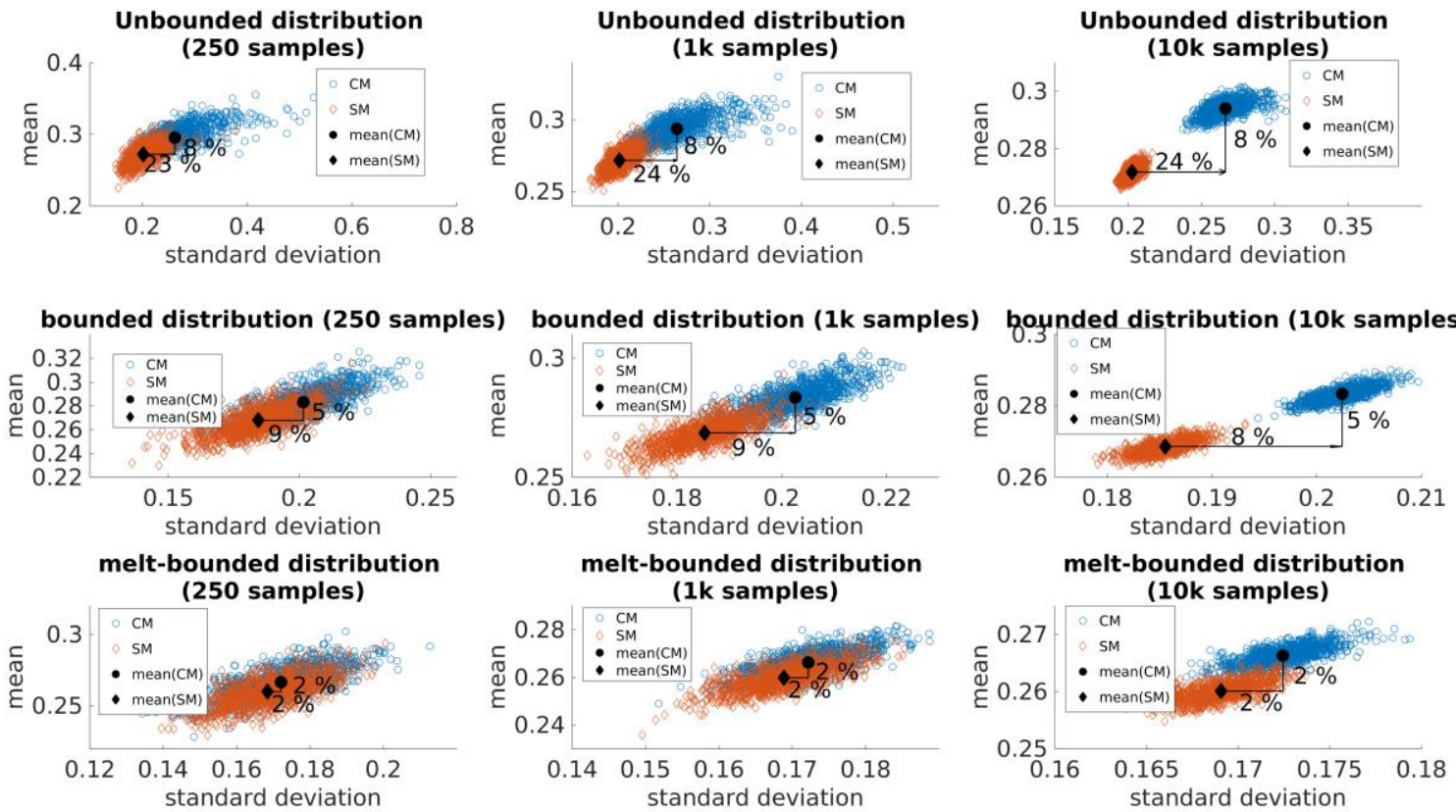

Fig. 13. Sensitivity analysis about the number of generated samples and the distribution type using the standard deviation-mean plots.

cumulative density function (CDF) of the theoretical law (Fig. 11 and 12), the resistance limit is defined with a significance level of 0.01. Note that using the PDF or the CDF, two

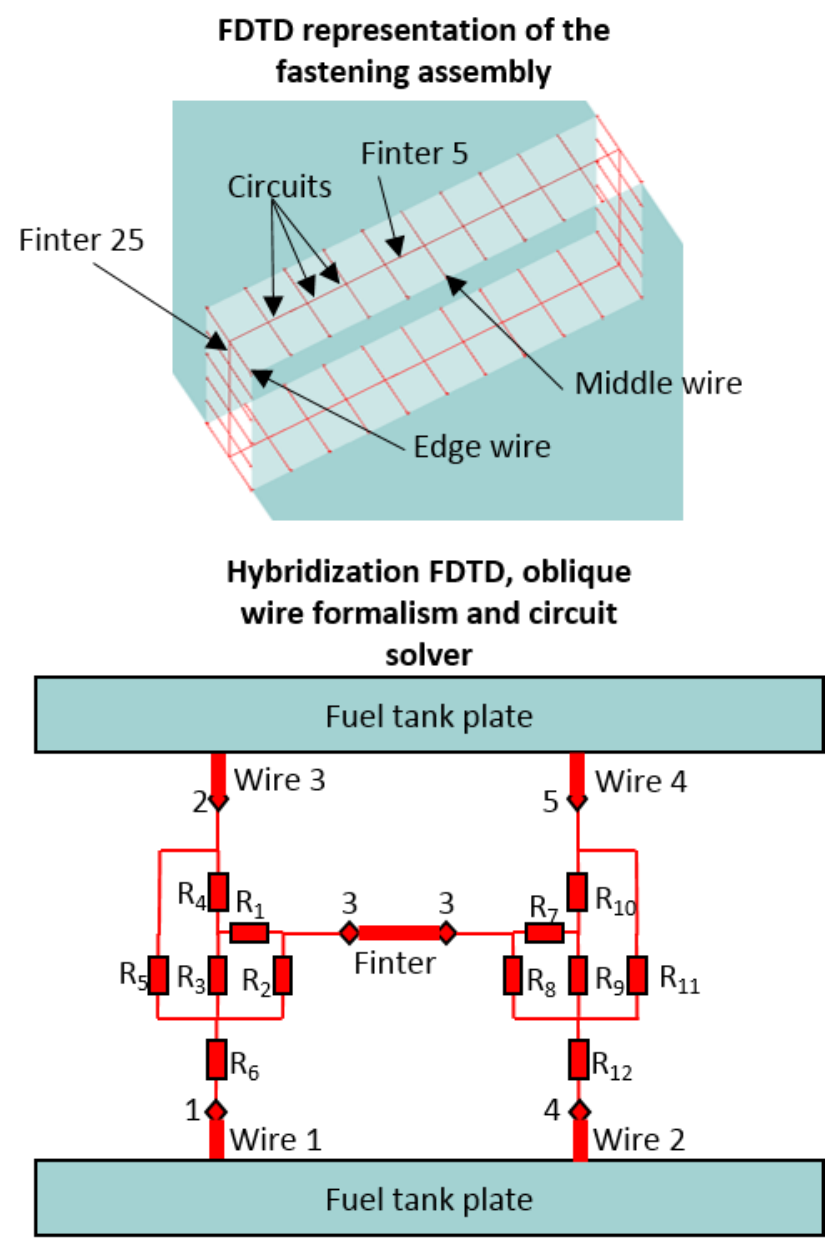

Fig. 14. Fastening assembly modelling with float nut fastener. different limits are obtained even if they are so closed. It is due to the two different methods used to determine the limits. Indeed, with the PDF, the well-known trapezoidal rule is used while the CDF give directly the limit. The upper bound is chosen according to $\mathrm{CDF}$, i.e. the resistances cannot outstripped $105 \mathrm{~m} \Omega$. Hence, generated resistances outstripped the limit are fixed either at the limit (bounded distribution) or around the mean (melt-bounded distribution). Fig. 13 presents the results for the three cases with 250, 1000 or 10000 generated samples. Each blue point represents the mean and standard deviation values for the $\mathrm{CM}$ samples from the optimized distribution generating, while the red ones are the values for the simple model (SM) used in the part I from the theoretical distribution generation. For each case, 1000 sets of samples are generated. The black diamond and the black point are respectively the mean of the scattered red diamonds and the scattered blue points. If clearly the mean and the standard deviation are not samples number dependent, the bounding condition and the outstripped samples redistribution have a major role. The melt-bounded distribution presents the better enhancement with only mean error of $2 \%$ on both indicators.

In practice, it is not easy to control the bounding condition for the optimized distribution which results to the equivalent resistance computation. The adopted trick is to reject and generate again the samples provided that the equivalent resistance outstrips the limit. Doing that, we mimic the meltbounded distribution.

\section{FUEL TANK MODELLING RESULTS}

\section{A. Fuel tank modelling}

The design of the fuel tank is the same as Fig. I-10. For the sake of brevity, in this paper we will present only the fastening assembly which is the only one to change in the FDTD modelling. The others parameters can be found in Section I-II. 
TABLE IV

STATISTICAL INDICATORS OF THE GENERATED SAMPLES FOR THE SM AND CM (ALL VALUES ARE GIVEN IN MILLIOHMS)

\begin{tabular}{|l|l|l|}
\cline { 2 - 3 } \multicolumn{1}{c|}{} & SM & CM \\
\hline Mean & 26.1 & 26.8 \\
\hline Standard deviation & 17.2 & 17.6 \\
\hline Maximum & 104.3 & 104.0 \\
\hline Minimum & 0.15 & 0.09 \\
\hline
\end{tabular}

The main difference between the SM and the $\mathrm{CM}$ is the connection between each fastener made with the CM. Fig. 14 presents the fastening assembly. A circuit of a float nut fastener is inserted at each node of the assembly middle line. Using the CM presented in Fig. 2 with the numbering system
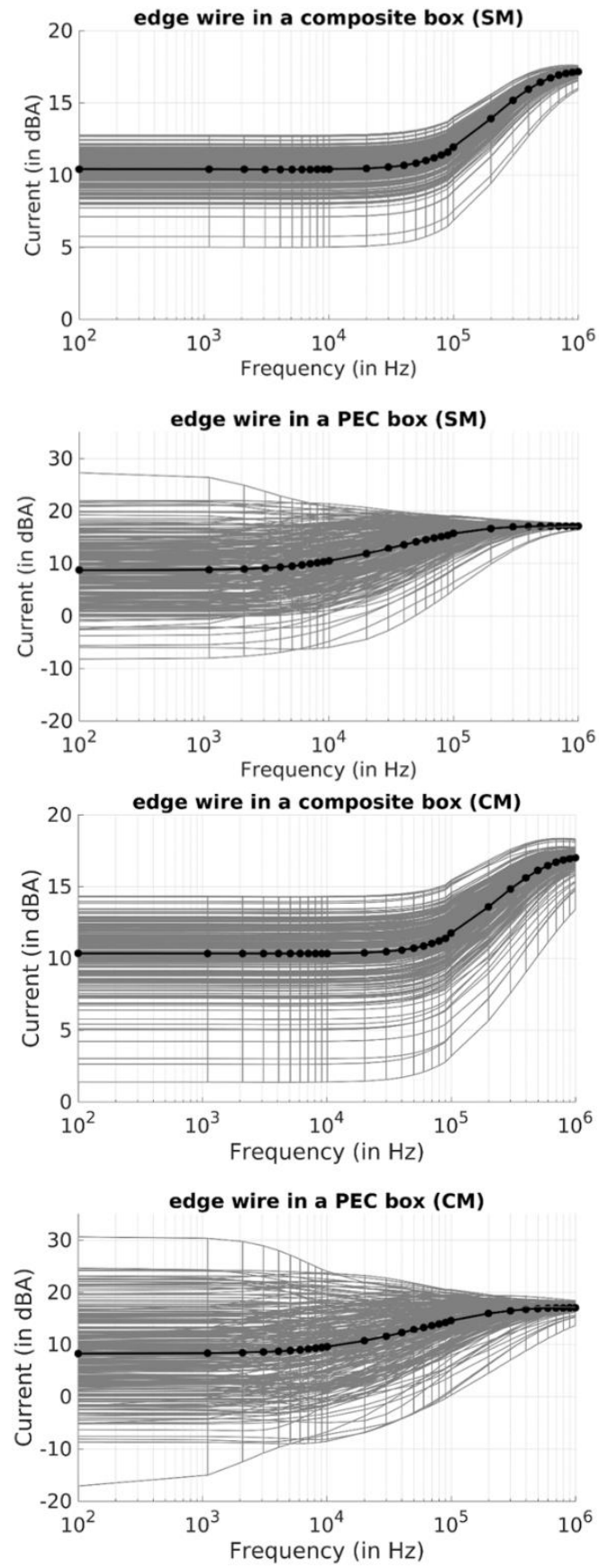

Fig. 15. Comparison between the SM and CM current distributions in the edge wire in function of frequency for a metallic and composite fuel tank. The black line with circle represents the mean of the 246 simulations (grey lines). of Table I and the hybridization method described in Fig. 5, the representation of two neighboring circuits is shown in Fig. 14. New current pathway are introduced by the CM with input wires (here noted 1 and 2), output wires (noted 3 and 4) and a wire between each circuit noted Finter. We note that each circuit is connected to its two neighboring circuits by a port 3 and a wire Finter. As a consequence, all circuits are connected themselves.

\section{B. Current distributions}

In order to be consistent with the part I, we compare the current distributions in the edge wire and the middle wire between the SM and the CM. As reminder, using the method
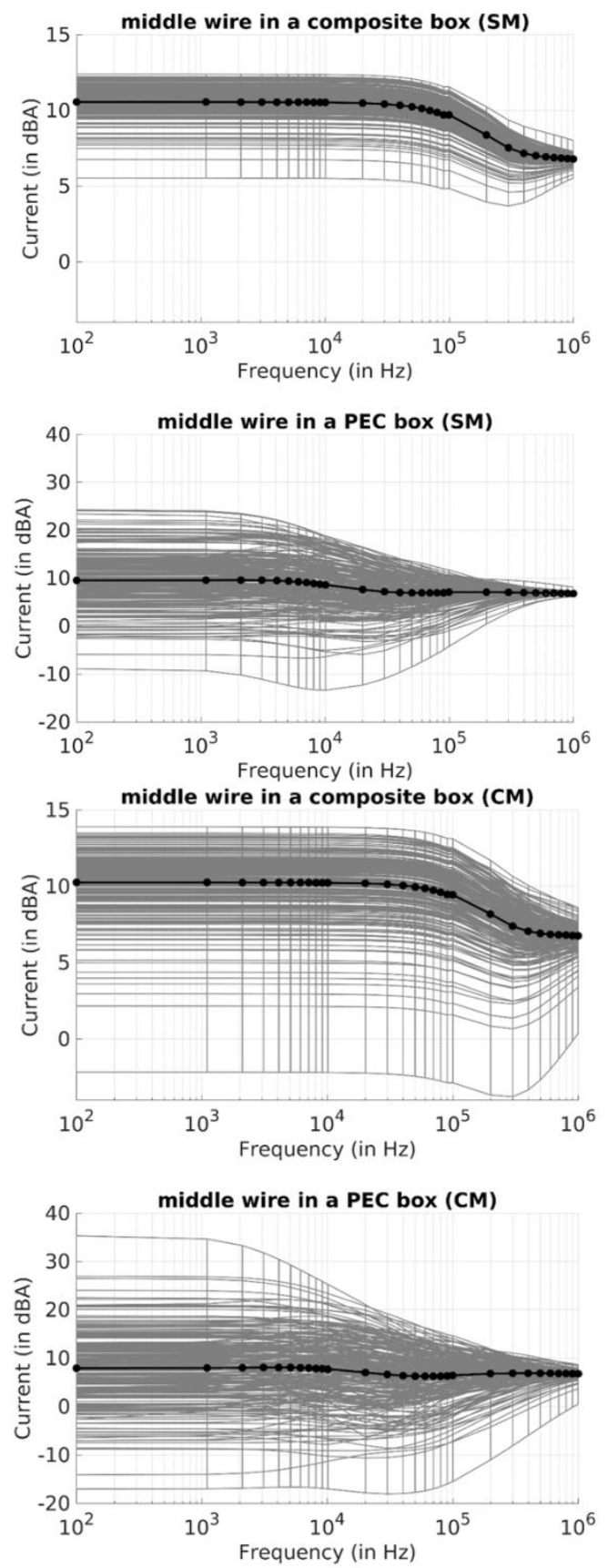

Fig. 16. Comparison between the SM and CM current distributions in the middle wire in function of frequency for a metallic and composite fuel tank. The black line with circle represents the mean of the 246 simulations (grey lines). 

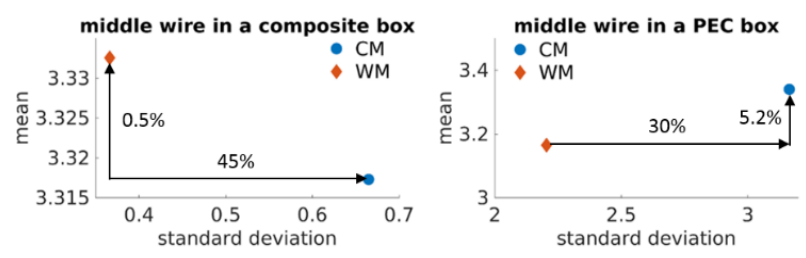

edge wire in a composite box
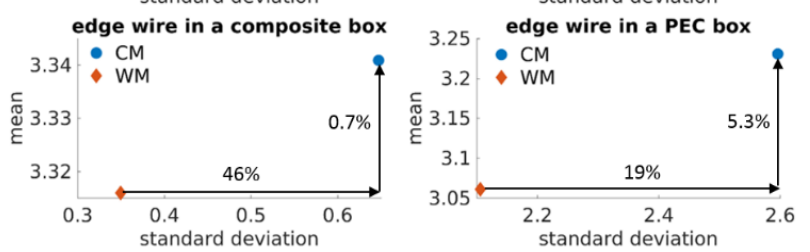

Fig. 17. Comparison of the means and the standard deviation of the SM and $\mathrm{CM}$ current distributions at $100 \mathrm{~Hz}$ for a metallic and composite fuel tank. The vectors values represent the relative error between both models.

presented in the section III, the generated samples aim for the same statistical behavior for both models. Some statistical indicators highlight this result in the Table IV. We decide to use the input wires for the current readings since the main interest is the incoming current in the fastener in order to establish the constraint. Fig. 15 and Fig. 16 present the current distributions respectively for the edge wire and the middle wire for a metallic fuel tank and a composite fuel tank using the SM or the CM. The black line with circle represents the mean of the 246 simulations (grey lines). Regarding these results, the SM and the CM seems to have a good agreement. As a consequence, concerning the impact of the type of material, the conclusions are the same as in part I highlighting the major role of the composite in the current distribution which limits the impact of the fastener resistances. Nevertheless, the current distributions with the CM seems to be more spread out. In particular, the current maximums are always higher for the CM than the SM. We note the same conclusion for the current minimums. Fig. 17 presents four mean-standard deviation plots computed with the 246 values
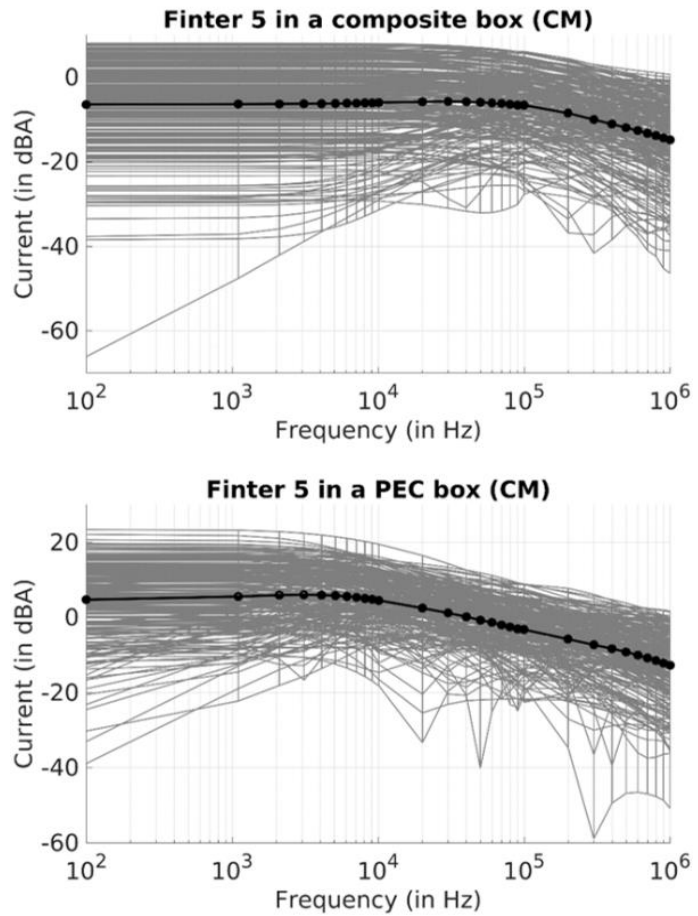

of current (in linear) at $100 \mathrm{~Hz}$. It confirms the discrepancy between the CM and SM standard deviations while the means are in agreement. The obvious conclusion is that the two models cannot be equivalent even if their equivalent resistances follow the same probability law. This difference is clearly due to the connections between each fastener in the CM. Indeed, Fig. 18 highlights the influence of these connections. Although for several simulations the currents flowing in these wires are small, they can have an important impact. As always, the impact in terms of current levels is more important for the metallic fuel tank than the composite one. The slope inversion of some current responses is due to an inversion of the current circulation. This can be explain by the fact than the inductive effect draining the current on the edge of the plate can be direction-opposed to the resistive effect when frequencies increase beyond several $\mathrm{kHz}$. Furthermore, we note that the wires closed to the edges (here Finter 25) have a large bandwidth impact contrary to the wires in the middle (Finter 5) since Finter 25 allows a current way to the edge fastener.

\section{V.CONCLUSION}

This paper presents a new FDTD model based on a full lumped resistive $\mathrm{CM}$ of the fastening assemblies that can be used in an aircraft EM modeling for improving the lightning protection and physical understanding of the phenomena. An original method dealing with DC measurement and an optimization process are presented in order to determine the numerical values of the lumped elements. This characterization method is really efficient provided that the number of measurements is greater than the number of lumped resistances and covers each node of the circuit. It is important to note that all the needed measurements can be made with this kind of fastening assemblies even if we have presented a numerical validation of this method with a simulated
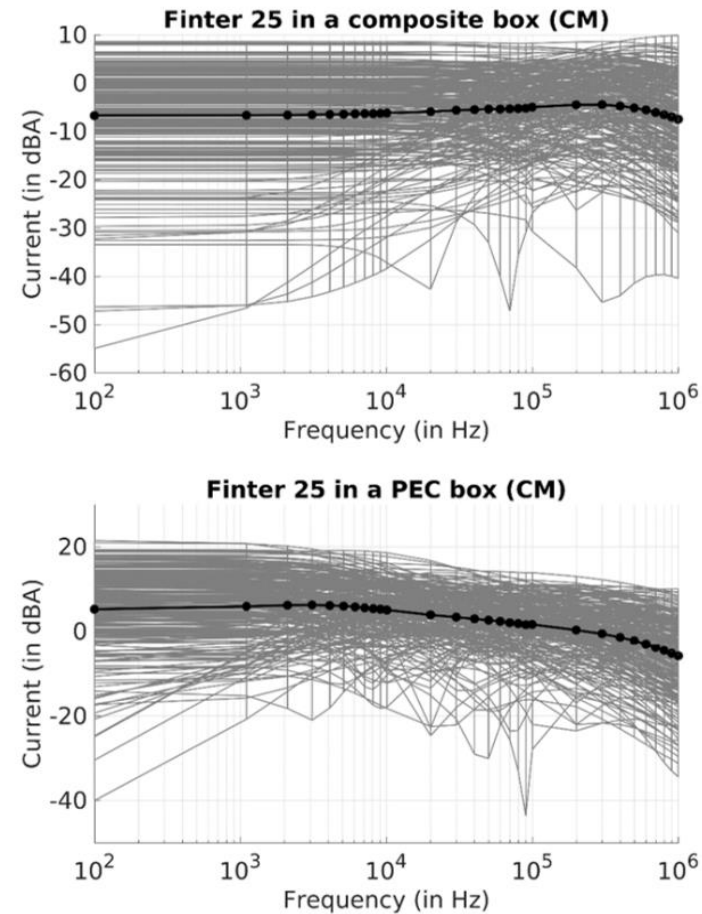

Fig. 18. Comparison between the SM and CM current distributions of two Finter wires located between two fasteners in function of frequency for a metallic and composite fuel tank. Finter 5 in the middle and Finter 25 at the edge of the tank 
measures. A sensitivity analysis about the measurement uncertainties has been performed. It highlights a strong sensitivity against measurement errors. Accurate measurement are required for an accurate characterization. The original implementation of the $\mathrm{CM}$ with the oblique thin wire formalism in the FDTD method is detailed. Notwithstanding the lack of lumped measures, a statistical model has been applied for each lumped elements of the CM and set up to recover the statistical law of the SM as established in [1]. This statistical model is based on a strong condition which is that all the lumped elements follow the same statistical law. Finally, a stochastic study about the fastening assembly on a metallic fuel tank and a composite one was carried out to quantify the current distributions. Comparison between SM and CM models is showed. As in [1], the aim is to overcome the fastener uncertainties and to estimate the extreme values of current. The results highlight the importance of modeling the direct link between adjacent fasteners which are not represented with the SM in [1]. CM model is also of great interest for direct lightning attachment on a fastener.

\section{REFERENCES}

[1]P. Monferran, C. Guiffaut, A. Reinei, F. Fustin, F. Tristant, "Lightning Effects on Fastening Assemblies of an Aircraft Fuel Tank, Part I: Statistical Method and Simple FDTD Modeling".

[2]I. Revel, A. Herve, G. Peres, M. Webster, R. Maddison, F. Flourens, "Undestanding of edge glow phenomenon," in ICOLSE, 2013.

[3]L. Chemartin, P. Lalande, F. Tristant. "Modeling and simulation of sparking in fastening assemblies," in ICOLSE, Seattle (WA), pp. 18-20, 2013.

[4] Advisory Circular AC20-53B - Protection of aircraft fuel systems against fuel vapor ignition caused by lightning - FAA, May 2006.

[5]J.A Plumer, J.D Robb, "The direct effects of lightning on aircraft," IEEE Trans. Electromagn. Compat., vol. EMC-24, no. 2, pp. 158-172, 1982.

[6]B. Lepetit, I. Revel, G. Peres, L. Andrivet, F. Flourens, "In-strike dynamical measurements of contact resistances," in ICLP, Cagliari, Italy, 2010.

[7]J.H. Covey, “Arc suppression around fasteners", Patent No. US4905931, 2 Jan. 1990.

[8]S. Evans, I. Revel, M. Cole, R. Mills, "Lightning Strike Protection of Aircraft Structural Joints," in ICLP, 2014.

[9]H. Mulazimoglu, L. Haylock, "Recent Developments in Techniques to Minimize Lightning Current Arcing Between Fasteners and Composites Structure," in ICOLSE, 2011.

[10]Chemartin, P. Lalande, B. Peyrou, A. Chazottes, P.Q. Elias, C. Delalondre, B.G. Cheron, F. Lago, "Direct Effects of Lightning on Aircraft Structure: Analysis of the Thermal, Electrical and Mechanical Constraints,"AerospaceLab, Issue 5, 2012.

[11]Time ElectroMagnetic Simulator - Finite Difference software, TEMSIFD, CNRS, Univ. of Limoges, Limoges, France, 2006.

[12]C. Guiffaut, A. Reineix, B. Pecqueux, "New oblique thin wire formalism in the FDTD method with multiwire junctions," IEEE Trans. Antennas Propag., vol. 60, no. 3, pp. 1458-1466, 2012.

[13]F. Tristant, F. Fustin, F. Terrade, M. Roussel, F. Parfait, F. Lago, M. Berthet, A. Laisné, C. Guiffaut, and A. Reineix, "Fuel tank safety - 3D computations for attachment and conduction assessment," in ICOLSE, Toulouse, France, Sep. 2015.

[14]C. Weber, D. Lalonde, W. Tse, S. Brault, F. Ahmad, J. Kitaygorsky, "Lightning response of a composite wing test box : a validation of simulation results," in ICOLSE, Toulouse, France, Sep. 2015

[15]OM16 milliohmmeter / microhmmeter Datasheet, http://www.aoip.fr/product/micro-ohmmetres/om-16/. AOIP, BP 182, 91133 RIS ORANGIS CEDEX France

[16]L. Brancik, "Programs for fast numerical inversion of Laplace tranforms in matlab language environment". Institute of Theoretical and Experimental Electrical Engineering, Faculty of Electrical Engineering and Computer Science, Brno University of Technology.
[17] J. E Dennis, D. M. Gay, R. E Welsch, "An Adaptive Nonlinear Least Squares Algorithm," ACM Trans. Math. Software 7, pp. 348-368 and 369-383, 1981.

[18]D. M. Gay, "A Trust Region Approach to Linearly Constrained Optimization in Numerical Analysis," Proceedings, Dundee (1983), edited by D. F. Griffiths, Lecture Notes in Mathematics, Springer-Verlag, Berlin, Heidelberg, New York, (1984).

[19]E. Portes dos Santos, C. Ribeiro Xavier, P. Goldfeld, F. Dicksteir, R. Weber dos Santos, "Comparing Genetic Algorithms and Newton-Like Methods for the Solution of the History Matching Problem," Computational Science-ICCS 2009, vol. 5544, pp. 377-386, 2009.

[20]P. A. Fox, A. P. Hall, and N. L. Schryer, "The PORT mathematical subroutine library," ACM Trans. Math. Software, vol. 4, no. 2, pp. 104126, June 1978.

[21] Holger Vogt, Marcel Hendrix, Paolo Nenzi, "Ngspice Users Manual Version 27plus (Describes ngspice master branch version)" [Online]. Available : http://ngspice.sourceforge.net/docs/ngspice-manual.pdf

[22]Ho, Ruehli, and Brennan, "The Modified Nodal Approach to Network Analysis," in Proc. 1974 Int. Symposium on Circuits and Systems, San Francisco, pp. 505-509.

[23]E. J. Davison, "A high-order Crank-Nicholson technique for solving differential equations," Comput. J., vol. 10, pp. 195-197, 1967.

[24]F. Tristant, F. Fustin, "Lightning direct effects degradation of composite material depending on paint thickness," in ICOLSE, Nagoya, Japan, 2017.

[25]A. Bigand, Y. Duval, "Quantification of the mechanical impact of lightning strike protection explosion confined by thick paint," in ICOLSE, Nagoya, Japan, 2017.

Biographies of the other co-authors are currently not available. 